\title{
ON DIRECT PRODUCTS OF REGULAR $p$-GROUPS
}

\author{
J. R. J. GROVES
}

ABSTRACT. We prove that, for each prime $p$, there exists a regular $p$-group $H(p)$ with the property that, if $G$ is a regular $p$-group and $G \times H(p)$ is regular, then the derived group of $G$ has exponent $p$. This provides a strong converse to a theorem of Grün.

Introduction. It has long been known that the direct product of regular p-groups need not be regular. The first example was due to $\mathrm{H}$. Wielandt and may be found in [2, III, 10.3]. In the positive direction, however, Grün [3] has shown that if $G$ is a regular $p$-group whose derived group has exponent $p$, then $G \times H$ is regular for every regular $p$-group $H$. The purpose of this note is to prove a strong converse to this theorem. We will prove:

THEOREM. For each prime $p$, there exists a regular p-group $H(p)$ with the following property:

If $G$ is a regular p-group and $G \times H(p)$ is also regular, then the derived group of $G$ has exponent dividing $p$.

We recall that a finite $p$-group $G$ is regular, if, whenever $g, h \in G$, there exists an element $d$ of the derived group of the group generated by $g$ and $h$ such that $(g h)^{p}=g^{p} h^{p} d^{p}$. If, in addition, $G^{\prime}$ has exponent $p$, then $(g h)^{p}=$ $g^{p} h^{p}$ for all $g, h$ belonging to $G$ and so $G$ is $p$-abelian in the sense of Baer [1]. Conversely, if $G$ is $p$-abelian, then $G$ is evidently regular and it follows from standard results on regular groups (see, for example, [2, III, 10]), that $G^{\prime}$ has exponent dividing $p$. Hence, recalling the quoted theorem of Grün, we have

COROllary. Let $G$ be a regular p-group. Then $G \times H$ is regular, for each regular p-group $H$, if and only if $G$ is p-abelian.

As regular 2-groups are abelian, the Theorem is trivial if $p=2$ and so we will henceforth assume that $p$ is an odd prime. We denote the commutator $g^{-1} h^{-1} g h$ of elements $g$ and $h$ of a group $G$ by $[g, h]$-and similarly for higher commutators-and the derived group of $G$ by $G^{\prime}$. Also, $(g, h)$ will denote an element of the direct product $G \times H$, where $g \in G$ and $h \in H$. All groups considered in this note are finite.

Received by the editors June 6, 1972.

AMS (MOS) subject classifications (1970). Primary 20D15; Secondary 20D40.

Key words and phrases. Finite $p$-group, regular, direct product, $p$-abelian.

(C) American Mathematical Society 1973 
Proof of the Theorem. We begin by constructing the group $H(p)$. We have some freedom of choice in this; in fact it will suffice that our group have the following properties:

(i) $H(p)$ can be generated by two elements $a$ and $b$.

(ii) $H(p)$ is regular but not $p$-abelian.

(iii) Every commutator, in $H(p)$, of weight 3 or more has order dividing p.

(iv) $(a b)^{p}=a^{p} b^{p}$ and $[a, b]^{p} \neq 1$.

Our construction is essentially due to Paul M. Weichsel [4], who proves a similar theorem under the restriction that $G$ be metabelian. We repeat it here largely for convenience (and because it involves a slight twist on that construction), but refer to [4] for fuller details. Let $A$ denote the direct product $\left\langle a_{1}\right\rangle \times\left\langle a_{2}\right\rangle \times \cdots \times\left\langle a_{p-1}\right\rangle$, where $\left\langle a_{i}\right\rangle$ is a cyclic group, of order $p^{2}$ if $i=1$ or 2 and of order $p$ if $i \geqq 3$. Let $t$ denote $(p-1) / 2$ and let $b$ denote the automorphism of $A$ defined by:

$$
\begin{aligned}
& b: a_{i} \rightarrow a_{i} a_{i+1} \quad(1 \leqq i<p-1), \\
& b: a_{p-1} \rightarrow a_{p-1} a_{2}^{p t} .
\end{aligned}
$$

Then the group, $H(p)$, that we require, is the split extension of $A$ by $\langle b\rangle$. It can be verified, either by direct calculation or by reference to [4], that $b$ is, in fact, an automorphism of $A$ and that the group $H(p)$ satisfies the required conditions, with $a_{1}=a$. In particular, property (iv) is verified as follows:

$$
(a b)^{p}=a^{p} b^{p}[b, a]^{p t}\left[a, \underset{(p-1 \text { times })}{, \cdots, b]}=a^{p} b^{p}\right.
$$

since $[a, b, \cdots, b]=[a, b]^{p t}$.

Let $G$ be a $p$-group which is regular but not $p$-abelian; we will show that $G \times H(p)$ is irregular. It suffices to assume that every proper subgroup and every proper homomorphic image of $G$ is $p$-abelian-for, otherwise, we could take a section of $G$ with these properties. We will now extract a few relevant properties of $G$.

As $G$ is not $p$-abelian, there exist elements $g$ and $h$ of $G$ such that $(g h)^{p} \neq$ $g^{p} h^{p}$ and, by the minimality of $G$, these must generate $G$. Let $M$ be a central subgroup of order $p$. By the minimality of $G, G \mid M$ is $p$-abelian and so $G^{\prime} \mid M$ has exponent $p$. Thus, if $g_{1}, h_{1}$ and $k$ are arbitrary elements of $G,\left[g_{1}, h_{1}\right]^{p} \in$ $M$ and so $\left[\left[g_{1}, h_{1}\right]^{p}, k\right]=1$. Hence, by a standard result on regular groups $\left[2\right.$, III, 10.6], $\left[g_{1}, h_{1}, k\right]^{p}=1$ and so every commutator of weight 3 or more has order dividing $p$. Thus, as $G$ is regular, $(g h)^{p}=g^{p} h^{p}[g, h]^{p r}$, for some integer $r$. But $[g, h]^{p} \in M$ and so, as $(g h)^{p} \neq g^{p} h^{p},[g, h]$ has order precisely $p^{2}$ and $r$ is prime to $p$.

The proof of the theorem now follows very quickly. For, suppose that $G \times H(p)$ were regular, and let $x=(g, a)$ and $y=(h, b)$. As $G$ and $H(p)$ 
both have the property that commutators of weight 3 or more have order dividing $p, G \times H(p)$ also has this property. Hence $(x y)^{p}=x^{p} y^{p}[x, y]^{p s}$ for some integer $s$. But,

Thus,

$$
\begin{aligned}
(x y)^{p} & =(g h, a b)^{p}=\left((g h)^{p},(a b)^{p}\right) \\
& =\left(g^{p} h^{p}[g, h]^{p r}, a^{p} b^{p}\right)=x^{p} y^{p}\left([g, h]^{p r}, 1\right) .
\end{aligned}
$$

$$
\left([g, h]^{p r}, 1\right)=[x, y]^{p s}=\left([g, h]^{p s},[a, b]^{p s}\right),
$$

and so $[g, h]^{p r}=[g, h]^{p s}$ and $[a, b]^{p s}=1$. But $[a, b]^{p} \neq 1$ and therefore $p \mid s$. It follows that $[g, h]^{p r}=1-$ a contradiction which completes the proof of the theorem.

\section{REFERENCES}

1. Reinhold Baer, Factorisation of n-soluble and n-nilpotent groups, Proc. Amer. Math. Soc. 4 (1953), 15-16. MR 14, 722.

2. B. Huppert, Endliche Gruppen. 1, Die Grundlehren der math. Wissenschaften, Band 134, Springer-Verlag, Berlin and New York, 1967. MR 37 \#302.

3. Otto Grün, Uber das direkte Produkt regulärer p-Gruppen, Arch. Math. 5 (1954), 241-243. MR 15, 852.

4. Paul M. Weichsel, Regular p-groups and varieties, Math. Z. 95 (1967), 223-231. MR 34 \#4364.

Department of Mathematics, University of Manitoba, Winnipeg, Manitoba, CANADA

Current address: Department of Mathematics, University of Melbourne, Parkville, Victoria, Australia 